\title{
EPISTEMOLOGICAL CONSIDERATIONS of GALILEO'S METHOD
}

II:AN C:AMPBEI.I. New School for Social Research

One of the themes in the discussion about Galileo's work is the debate about the extent to which he is an empiricist, or whether he is seen primarily as a rationalist. More specifically we can focus on determining what sort of an empiricist he is, how he uses mathematics, and how this reflects his philosophy of nature. Without attempting to definitively characterize him as either rationalist or empiricist, this paper considers the manner in which Galileo's conclusions rest on what may be identified as rational or empirical grounds. As will be shown, the strains of his empiricism are various and relative to the types of phenomenon investigated by him. Further, to the modern reader, a consideration of his work also presents the epistemologically interesting problem of recognizing theoretical foundational achievements in their genetical stages, after living in an age when the puzzles he tackled now have adequate theoretical description, or are no longer physically inaccessible as they were to him (such as the earth's motion). Our present investigation, therefore, is guided by an interest in the epistemological 'fault' which Galileo's work represents. Because his concerns were with Nature, the present inquiry is directed not only to the character of knowing, but to the character of mathematical and natural necessity as well.

To examine these questions it is helpful to start in the context of the observations stillman Drake provides about Galileo's self-image in section xxVI of his introduction to "The Assayer"[1]. There he refers to Galileo's odd insistance of having the title 'philosopher' in addition to 'court mathematician' and remarks that

He agreed [with Telesio] that in order to become science, philosophy must throw out blind respect for authority, but he also saw that neither observation, nor reasoning, nor the use of mathematics could be thrown out along with this. 
True philosophy had to be built upon the interplay of all three, and no combination could supply the absence of any one of them. [2]

Further, that "his entire attitude has been well summed up by the saying that he was never willing to accept any intermediary between himself and nature. "[3]

"The difference between philosophizing and studying philosophy," wrote Galileo in his notes on Lagalla's book, "is that which exists between drawing from nature and copying pictures. In order to become accustomed to handling the pen or crayon in good style, it is right to begin by redrawing good pictures created by excellent artists. Likewise in order to stimulate the mind and guide it toward good philosophy, it is useful to observe the things that have already been investigated by others in their philosophizing; especially those which are true and certain, these being chiefly mathematical .... But men who go on forever copying pictures and never get around to drawing from nature can never become perfect artists, or even good judges of painting. For they remain unpracticed in separating the good from the bad, or the accurately from the poorly drawn, by means of recognizing in nature itself (as a result of countless experiences) the true effects of foreshortening, of backgrounds, of lights and shadows, of reflections, and of the infinite variations in differing viewpoints... . In the same way a man will never become a philosopher by worrying forever about the writings of other men, without ever raising his own eyes to nature's works in the attempt to recognize there the truths already known and to investigate some of the infinite number that remain to be discovered. This, I say, will never make a man a philosopher, but only a student of other philosophers and an expert in their works. "[4]

Notwithstanding the obvious mediation we must apply as readers and discussants of his work, these passages help us assume a similar posture towards Galileo, that is, one without intermediaries. To get a sense of what he described and how he did it, it helps to brush aside all our conceptions of modern physical theory, experimental techniques, and technology through which our understanding of the physical universe is as much boggled and dissipated as it is mediated and extended. 
What we should picture is a man well-versed in the mathematics up to his day, especially the works of Archimedes and Euclid, who was equipped primarily with a powerful intellect, and who set out to describe, among other things, the motion in the heavens and of the objects found at hand around him.

Before we turn to a direct examination of some features of his work in the Dialogue on the Great World systems and Two New Sciences, I, $\frac{d}{1 i k e}$ to discuss what's at issue in the discussion of his 'empiricism' or his 'rationalism' or even 'platonism'. strictly speaking, of course, questions about the structure of knowledge itself, its origination from experience or thought, and what sort of certainty it has were not the main topics of his investigations. clearly, the polemic of the Dialogue centers on the motion of the earth and its position in the universe. In Two New sciences is presented the consideration of cohesion in solid bodies and their motion on the earth. It is we, looking bäck on his work, who try to determine how questions of this nature can be answered and on what its status as knowledge depends. The question we ask ourselves is: what is involved in being able to say from our vantage point of walking on its surface that the earth moves? or what sort of description can we make of the motion of a body falling to the ground? The extent to which these descriptions rest on what can be observed or on how we make sense of it correlates with the extent of our empiricism or rationalism.

Within this context we begin to see that the empiricism of Galileo's conclusion that the earth moves, for example, is necessarily oblique. Lacking any sort of rocket technology, there is simply no point to which he could remove himself to make an observation, nor any direct way such a statement could be verified or experienced. What collectible information there is from the earth's surface has to be 'reasoned' to its status as evidence for its motion. For example, even the use of a telescope and its power to bring distant objects closer depends on the understanding that this enables us to better discern features which indeed belong to the object and are not due to some optical trick. Then, further, a reasoned framework has to be supplied, within which the properties of these distant bodies might be seen to have something to do with the problem at hand. The type of experiment and empirical evidence that can be employed in the determination of the earth's motion depends irreducibly on placement within some larger framework which has other bases (observable and/or theoretical) for its determination than the motion of the earth, on analogy of its motion to other observable motions, either in the sky or on 
the earth, or on hypotheses which are able to link observable phenomena with the earth's motion. This is a different sort of empiricism than that which bore out Mendel's hypothesis about the genetics of sweet peas, for example, where he was able to breed a control group and then simply observe if what he expected took place or not. When we fully appreciate the special difficulties of the determination of the earth's motion, albeit we see it fully within the realm of physical phenomena, we are not so unsympathetic to Cardinal Bellarmine's judgement of the evidence as ultimately no more than support for a working hypothesis. At the same time, we can see the extent to which Galileo's method was necessarily philosophical and how far our capability presently to so remove ourselves from the earth's surface as to be able to watch it move rests on essentially speculative maneuvers.

Apart from the strictly epistemological consideration, we can distinguish generally the use of experiment and evidence from the senses to which, of course, Galileo makes appeal numerous times. In so far as he is describing Nature, this realm necessarily provides what is to be taken up. As in his treatment of naturally accelerated motion, this is the actual motion of heavy bodies close to the earth's surface. A determination of which experiments among the many he describes were actually performed or not, we leave to the historians of science. Sometimes we are clearly asked to hypothesize a given situation. other times, as in the measure of the speed of a ball rolling down a grooved plank with a water clock, they are presented precisely to illustrate how his theorizing truly describes what happens. The empiricism of this case is not so much a determination of the validity of Galileo's analysis; the theory already has a validity from its being derived mathematically. Here it is used to insure the correspondence of the two--mathematical description and the occurrence in nature.

The main point of this discussion has been to set off the strictly epistemological question which pertains to the structure of knowing itself, from the different senses of 'empiricism' and its relativity to what it is summoned to establish and to what is establishable empirically. We have seen generally that with respect to the determination of the earth's motion, substantiation rests irreducibly on theoretical grounds. For the description of naturally accelerated motion, on the other hand, the progress of a ball down an incline or the free fall of an object to the earth is entirely observable. This is not to deny a dimension of the analysis which is essentially theoretical, too, but simply to distinguish its type of empiricism. 
We will now first take up some of the arguments in the Dialogue on the Great World Systems and then turn to the analysis on Local Motion in the Two New sciences to more clearly demonstrate this difference.

The first issue which is addressed in the Dialogue is the Aristotelean separation of realms--celestial and earthly. The immobility of the earth rested in part on its opposition to the divine. The earth is susceptible to generation, corruptibility, and alteration. The motion natural to it is upwards and downwards. The heavens are eternal, changeless, and perfect. Circular motion is complete and self-contained and appropriate to the perfection of the celestial bodies. The earth's position at the center of the universe derives from the Aristotelean analysis of the three motions--motion is either up, away from the center; down, towards the center; or motion around the center. The motion of bodies on the earth being up and down, it follows that the earth is a center point.

Galileo refutes this position first by separating the consideration of corruptibility, generation etc. from that of a body's motion, saying that the former has nothing to do with the latter and secondly that these so-called terrestial characteristics can in fact also be found in the heavens. He points to the observance of sunspots to show that the sun sustains changes. From the property of spherical bodies of being more luminous when the surface is rough and can scatter the light than when they are perfectly smooth, he shows the moon's surface to have mountains and valleys, i.e., imperfections, and so to resemble the earth. The empirical evidence here is used with respect to the placement of the earth's motion within a theoretical framework, except negatively. In other words, because the earth's immobility derives from its place within the Aristotelean world picture, Galileo's first tack is to find flaws in the framework. Being able to show that the earth could have a place in the heavens along with the other celestial bodies weakens considerably the integrity of Aristotle's cosmology. and makes logical 'room' for the construction of another framework within which its motion would be possible.

Argument from observed motions as analogous to earth's is used both to refute its mobility (Aristotle's use) and to bolster the copernican system (Galileo's use). In the first case Aristotle maintains as follows:

All movables that move circularly seem to stay behind and move with more than one motion, except the first sphere (i.e., the Primum Mobile). 
Therefore the Earth moving about its own centre, being placed in the middle, ought of necessity to be carried about in two ways and also to stay behind. But, if this were so, it would follow that there should be a variation in the rising and setting of the fixed stars, as we do not perceive this to be done; therefore, the Earth does not move, etc. [5]

Aside from the retrograde motion, which of course would only be a consequence of observing the earth from the point of view of another planet, the double motion of the earth is precisely what Galileo would like to show. That such a motion might imply a variation of rising and setting of the stars which is lacking is an important consideration which he deals with at length later. Here he undercuts Aristotle's conclusion by showing its necessity is tied to the simple plurality of the motions. To show that it is stationary, he would also have to establish the impossibility of it moving with only one motion.

Galileo himself argues on analogy from his observation of a sphere floating in a bowl of water. In "The Assayer" he describes the spontaneous motion about its vertical axis that the sphere acquires relative to the bowl when the bowl is moved in a circle at arm's length. This was used to lend plausibility to the motion of the earth contrary to all other celestial motions that the copernican system introduced, and for which it was criticized as entailing something unlikely. Its utility is, of course, dependent on assumptions about the fluidity of the medium in which the earth is suspended, and in fact isn't helpful in the determination of the actual period of revolution about earth's axis. This only illustrates further the inconclusiveness of any particular empirical evidence in isolation from a framework which can connect the phenomenon with the earth's motion.

The third use of empirical evidence is that which is linked to the earth's motion by hypothesis. Such arguments as the straight fall of an object dropped from a tower, the lack of high winds, the stability of the objects on its surface, the lack of parallax of the fixed stars, the seasonal variations, and the tidal theory all fall generally in this class. Within this loose association we quickly find differences in their respective utility and purpose. However, they still have this in common, that all these observations having anything to do with the earth's motion clearly rest with their analysis. In regards to the possibility of making experiments which "demonstrate" such phenomena, Salviati sarcastically recommends to simplicius that he 

'have the experience' and suggests the following
'experiment'|6|:

- For a final proof of the nullity of all the experiments before alleged, I conceive it now a convenient time and place to demonstrate a way how to make an exact trial of them all. shut yourself up with some friend in the largest room below decks of some large ship and there procure gnats, flies, and such other small winged creatures. Also get a great tub full of water and within it put certain fishes; let also a certain bottle be hung up which, drop by drop, lets forth its water into another narrow-necked bottle placed underneath. Then, the ship lying still, observe how those small winged animals fly with like velocity towards all parts of the room; how the fishes swim indifferently towards all sides; and how the distilling drops all fall into the bottle placed underneath. And casting anything towards your friend, you need not throw it with more force one way than another, provided the distances be equal; and jumping broad, you will reach as far one way as another. Having observed all these particulars, though no man doubts that, so long as the vessel stands still, they ought to take place in this manner, make the ship move with what velocity you please, so long as the motion is uniform and not fluctuating this way and that. You shall not be able to discern the least alteration in all the forenamed effects, nor can you gather by any of them whether the ship moves or stands still... . [7]

Now that we are clearly in the realm of theoretical analysis we can begin to sketch out some of Galileo's method in this respect. The first task is to sort out the connection of the description of falling bodies to the motion of the earth. Insofar as the problem is formulated in Aristotelean terms, the main issue is the description of the path. Simplicius holds that on the evidence of the senses, one can see plainly with the eye that when the stone falls, it falls along the side of the tower perpendicularly, no deviating from side to side, and it lands at the base of the tower. If the earth moved, the tower would have displaced that amount from where it stood at the time of the release of the stone, leaving it behind. Analytically he is confined to seeing this as evidence of the earth's immobility. The three motions--up, down, and around the center-were exhaustive of those naturally occurring; and the stability of the center point relative to these motions 
is necessarily manifest. The completeness and finitude of the universe, however, rest on the understanding that this is the only center point for the motions in the heavens, that this center belongs accidentally to the earth, but absolutely to the universe. The first step out of this position is to conceive of multiple center points with circular motion about those occurring naturally. The move consists in being able to abstract from what is observationally available, in order to construct something which is conceptually available and indeed capable of telling us 'more' about any given phenomenon than what we can experience about it. That abstraction of this sort was possible and still applicable to naturally occurring phenomena was of course what was unacceptable to Aristotle in his separation of mathematics and physics. The mathematization of nature rests on the capability of constructions of the imagination to sometimes correspond with events which happen totally outside of it. [8] A kind of commensurability between the necessity involved in mathematical progressions and proof structures and the necessity to be found in a regularly occurring event must be grasped, or at least imagined possible. For Aristotle, the possibility of such a correlation was inconceivable. Hintikka and Remes[9] see this difference as a reflection of the choice of methodological paradigm. For Archimedes and Galileo, the structures of geometrical analysis were exemplary of what was to be discovered in phenomena. For Aristotle, things of motion, change, and animation followed more closely the model of human deliberation. At any rate, it is abstraction of the former sort that allows Galileo to make connections between the behavior of observable moving bodies and the hypothesized motion of the earth, which was unobservable.

The verifiability of such relationships, that is, relationships that are mathematical in the sense that through conception they provide a basis for further description which is inaccessible to merely empirically grounded observation (the epistemological correlate to this is the subject/object oppositional paradigm for knowing or any theory of consciousness which is based on the model of perception such as Husserlian phenomenology), is a separate issue, and any given analysis is subject to evaluation and critique along whatever lines possible. The description of a circular path for the ball which falls along the side of the tower, for example, wouldn't today be considered a 'correct' physical description of the falling body. Certainly Galileo himself did not think it definitive. As Santillana points out, he at times uses mathematical analyses that we know he knew were not the best 
descriptions of the phenomena in question. And others, such as his theory of the tides, were highly controversial and uncertain. It is indicative of his method throughout the Dialogue that these analyses were used essentially polemically--essentially to show that the carth's motion at least was not impossible.

We are now in a position to examine his use of mathematical analysis in the case of an observable movable. To do this I would like to focus on his treatment of naturally accelerated motion in Two New sciences. $[10]$ We must immediately recognize that the arguments and conclusions capable of being made are different in the following respect: that on the strength of his arguments alone, the strongest case that Galileo can make for the earth's motion is that it is not impossible that it does move. In contrast, the laws that he discovered concerning naturally accelerated motion can be considered to be positively asserted albeit with the degree of certitude that geometry can display. They are, however, ultimately similar in respect to the non-observability of the conclusions. Although we can watch the ball roll down the inclined plane, or fall from our hands to the ground, that the final degree of speed is twice that had it been carried over the same distance at constant velocity (Proposition I, Theorem I) or the proportion by which it accelerates as it does so (Proposition II, Theorem II, Corollary I), are neither of them ascertainable by simply watching what happens. In the case of the earth's motion, we were incapable of removing ourselves to a point of observation, giving rise to our dependency on what reason can tell us. In the latter, although the phenomenon itself is accessible, its measure is not, without the aid of the geometrical conceptual framework. By this we must necessarily attribute primacy to the role of reason in our ability to set up experiments which may confirm or refute our theoretical explorations and hunches. This at first sight neat separation, however, does not belie the somewhat miraculous correspondance that such reasoning seems to have with what actually naturally occurs. This connection is what sets Galileo's work off as a science of motion from an artifically conceived mathematical verity.

The entire set-up of the treatment of naturally accelerated motion is based on the definition of uniformly accelerated motion as that "which, abandoning rest, adds on to itself equal momenta of swiftness in equal times"[11] and the acceptance of his postulate that "the degrees of speed acquired by the same moveable over different inclinations of planes are equal whenever the heights of those planes are equal."[12] 
Although this cannot be proved, he provides us with the experiment of the ball swinging from a string around a nail to help persuade us that the impetus gained in descent is what determines the ball's subsequent ascent as it swings up past the nail which interrupts its arc. The rest of the propositions and theorems are constructed given a moveable which is uniformly accelerating. This is exemplary of the ultimately hypothetical character of any science. That a bali falling was uniformly accelerated was an idea that was accepted generally, although not really capable of being proven. All of the demonstrations depend on this as given, and once the science is worked out, its correspondence to actual occurrence is so compeiling (as analogous to the observations stillman Drake makes concerning Galileo's use of actual experiments in his work on projectile motion) we have no reason to reject it. The propositions and theorems establish certain properties of the motion, by way of working through different casses-- 1) straight along inclined planes and 2) circular motion--and the problems in the science are to make sure different geometrical constructions can be made according to what those properties would imply. His famous law on the eternality of unimpeded horizontal motion at constant velocity is not demonstrated, and is to be understood, and rightly so as he has placed it in a scholium[13] after the proposition (XXIII) which brings it to mind, apart from the rigorous structure of the rest of the science. [14] It is, however, strictly reasoned from the extrapolation of what would occur upon the removal of both downward acceleration and upward retardation. As he states it

- whatever degree of speed is found in the movable, this is by its nature [suapte natura] indelibly impressed on it when external causes of acceleration or retardation are removed, which occurs only on the horizontal plane; for on declining planes there is cause of more [maioris] acceleration, and on rising planes, of retardation. From this it likewise follows that motion in the horizontal is also eternal, since if it is indeed equable it is not even weakened or remitted, much less removed.[15]

What this paper has tried to do is reveal the epistemological structure of the arguments and method that Galileo used first in the Dialogue on the Great World Systems and secondly in Two New Sciences. Their similarities consist in their ultimate dependence on conceptualization, which emphasizes the strong 
'rationalism' to be found in Galileo. They are different in their use of both mathematical analysis and experiment. Because of the nature of the problem in the Dialogue, both different descriptions of motion of things on the earth and different empirical observations and experiments could only be used indirectly to support or refute the probability of the earth's motion. The conclusions of the Two New Sciences, in contrast, rest directly on the geometrical analysis of the phenomenon and experiments in this case are used to insure a natural correspondence. 
NOTES

${ }^{1}$ stillman Drake, translation Discoversies and Opinions of Galileo (New York: Double $\overline{d a y} \overline{A n c h o r}$ Books, 1957).

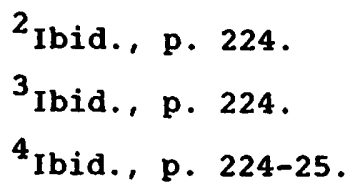

${ }^{5}$ Galilei Galileo, Dialogue on the Great World Systems, translated by $\mathbf{T}$. Salusbury, edited by Giorgio de Santillana (Chicago: University of Chicago Press, 1955), p. 63 .

'Another way of viewing the 'experiment' is to see it as the newtonian principle of relativity, that is, that it is possible to measure the acceleration of a coordinate system but not its velocity, in anecdotal form.

${ }^{7}$ Dialogue on the Great World Systems, p. 84 .

${ }^{8}$ If mathematics is understood primarily as a science of the imagination, the epistemological problem is how it can have applicability to something beyond the imagination.

${ }^{9} \mathrm{~J}$. Hintakka and $U$. Remes, The Methods of Analysis (Massachusetts: D. Reidel, 1974).

${ }^{10}$ Galilei Galileo, Two New Sciences, translated by stillman Drake (Madison, Wisconsin: University of Wisconsin Press, 1974).

${ }^{11}$ Ibid. , p. 154 .

12 Ibid. , p. 162 .

13 Ibid. , p. 197.

${ }^{14}$ This law which has the status of well-argued commentary in Galileo's treatise is equivalent to Newton's first principle.

${ }^{15}$ Two New Sciences, p. 197. 